\section{Tunable electrical memory characteristics by the morphology of self-assembled block copolymers:PCBM nanocomposite films $\dagger$}

\author{
Jhao-Cheng Chen, ${ }^{a}$ Cheng-Liang Liu, ${ }^{* b}$ Ya-Sen Sun, ${ }^{* c}$ Shih-Huang Tung ${ }^{d}$ and Wen-Chang Chen ${ }^{* a d}$
}

Received 25th August 2011, Accepted 11th October 2011

DOI: $10.1039 / \mathrm{c} 1 \mathrm{sm} 06622 \mathrm{f}$

\begin{abstract}
Resistive switchable memory devices were fabricated using self-assembled composite thin films of asymmetric poly(styrene-block-4-vinylpyridine) (PS-b-P4VP) block copolymers (BCP) and fullerene derivatives (PCBM). L1 (with a longer PS block) was comprised of discrete vertical P4VP nanocylinders embedded within the PS matrix whereas L2 (with a longer P4VP block) revealed a reverse morphology with a horizontal orientation. They were used to control the spatial location or distribution of the PCBM and the resultant memory characteristics. The devices with ITO/BCP:PCBM/ Al configurations exhibited variable multi-electronic characteristics, changing from insulating to bistable memory switching and highly conducting, as the PCBM content increased. The L1:PCBM memory device showed non-volatile write-once-read-many-times (WORM) memory behavior but the L2:PCBM device exhibited a volatile nature of static random access memory (SRAM). Both L1 and L2: PCBM composite devices revealed the improved switching performance upon solvent annealing procedures of the composite thin film. Our results suggested that the controlled morphology of the $\mathrm{BCP} / \mathrm{PCBM}$ composite could create nanoscale charge-storage elements for a high density memory device with a reduced bit cell size.
\end{abstract}

\section{Introduction}

Resistive switching materials made of organic polymer thin films have been recognized to be promising alternatives to their traditional inorganic counterparts due to the advantages of structural flexibility, low-cost, solution processability, and threedimensional stacking capabilities. ${ }^{1-7}$ The active layer exhibits distinct high and low conductance responses to applied voltages for application as digital memory devices with simple sandwiched structures. The reported polymer-based systems for such applications included conjugated polymers, ${ }^{8-10}$ functional polyimides (PIs), ${ }^{11-13}$ polymers with pendent electroactive chromophores, ${ }^{14-16}$ and polymeric composites. ${ }^{17-29}$

The memory switching behaviors of composite materials using various charge-storage filled additives (metal ${ }^{17-22}$ or fullerene derivatives ${ }^{23-29}$ ) in the supporting matrices were identified as

${ }^{a}$ Department of Chemical Engineering, National Taiwan University, Taipei, 10617, Taiwan. E-mail: chenwc@ntu.edu.tw; Tel: $+886-2-2362-8398$

${ }^{b}$ Department of Organic Device Engineering and Research Center for Organic Electronics, Yamagata University, Yonezawa, Yamagata, 9928510, Japan.E-mail: clliu@yz.yamagata-u.ac.jp; Tel: +81-238-26-3309

'Department of Chemical and Materials Engineering, National Central University, Taoyuan, 32001, Taiwan.E-mail: yssun@cc.ncu.edu.tw; Tel: +886-3-422-7151 ext 34205

${ }^{d}$ Institute of Polymer Science and Engineering, National Taiwan University, Taipei, 10617, Taiwan

$\dagger$ Electronic supplementary information (ESI) available: See DOI: $10.1039 / \mathrm{c} 1 \mathrm{sm} 06622 \mathrm{f}$ being achieved via several proposed mechanisms, such as charge trapping/detrapping, ${ }^{18,23,24}$ charge-transfer effect, ${ }^{17,21,22,27-29}$ filament formation, ${ }^{19,20}$ and polarization effect. ${ }^{25,26}$ When the recording domains are down to the nanoscale, new materials and advanced techniques must be explored to satisfy the need of storage capacity. However, the issues of non-homogenous largesize aggregated fillers within an active layer make it less possible to meet the requirements of scalable fabrication for the growing demand on high-density memory arrays of nanoscopic element cells. Block copolymers (BCP) can self-assemble into various ordered nanostructures (such as lamellae, cylinders, spheres and so on) with length scales ranging from 10 to $100 \mathrm{~nm}$ due to the repulsion between the chemically connected blocks. ${ }^{30-32}$ Recently, many efforts have been dedicated to achieving the spatial and orientational distribution of the nanoelements within the ordered scaffolds offered by BCP, because the cooperative self-assembly in polymeric hybrids offers rich possibilities to develop novel electrical properties. ${ }^{21,33-37}$ The diverse nanodomains of BCP bulk species can be achieved by tailoring the nature of BCP (chemical structures, the length of each block, and architecture) and micellar nanostructures of BCP films can be obtained by altering the polymer concentration, choice of solvent or processing conditions. ${ }^{30-32,38-46}$ Among the diverse nanostructures mentioned above, the spherical micelles of BCP were often selected as structural components for control over the functional clustering size and selective spatial location of filled additives in order to finely tune and optimize the memory properties. Leong et al first demonstrated the versatility of using 
self-assembled BCP with the in situ synthesis of Au nanoparticles (NPs) for polymer memory device fabrication, in which the capacitance-voltage $(C-V)$ or current-voltage $(I-V)$ hysteresis in the capacitor or transistor structure represented charge confinement in the embedded Au NPs. ${ }^{34-36}$ Besides the ordered cylindrical nanostructures of $\mathrm{BCP}$ being used to selectively sequester the Au NPs, they also exhibited resistive switching. ${ }^{21}$ The distribution of charge-storage sites within the thin memory film layer indeed determines the memory device performance. Recently, we demonstrated a supramolecular approach to prepare controlled domain sizes of poly(fluorenylstyrene)-blockpoly-(2-vinylpyridine) $\mathrm{P}(\mathrm{StFl}-b-\mathrm{P} 2 \mathrm{VP}): \mathrm{PCBM}$ composite thin films for use in a non-volatile memory device. ${ }^{28}$ However, the PCBM domain in the $\mathrm{P}(\mathrm{StFl})-b$-P2VP matrix was disordered and non-oriented. Thus, it would be of significant interest to explore the correlation between resistive switching of polymer composites and their self-assembled spatial ordered morphologies systematically.

In this paper, we report the morphology, properties, and memory device characteristics of self-assembled asymmetric poly (styrene-block-4-vinylpyridine) (PS- $b$-P4VP) BCP and their hybrids with different amounts of PCBM. This composite system served as an excellent archetype; the self-assembled BCP generated the nanotemplate to selectively control the spatial locations of the PCBM molecules as the electronic traps, in which the nitrogen atom in the P4VP block provided the physical stabilization for achieving uniform PCBM distribution. The chain length of each block and solvent annealing conditions could manipulate the PCBM aggregated nanostructures in the continuous insulating matrix that created tunneling barriers for charge-transport. Atomic force microscopy (AFM), transmission electron microscopy (TEM), and grazing-incident small angle X-ray scattering (GISAXS) were performed to characterize the morphology of the BCP composite thin films. The memory device with the configuration of ITO/BCP composite/Al was employed to explore the energy levels of the memory characteristics that were further analyzed by the appropriate conduction model. The self-organized PCBM approach created the nanotrapping sites obtained through the BCM morphology that determined the memory characteristics.

\section{Experimental section}

\subsection{Materials}

Two asymmetric linear poly(styrene-block-4-vinylpyridine) (PS- $b$ P4VP) diblock copolymers were purchased from Polymer Source Inc. (Dorval, Canada) which were named $\mathbf{L 1}\left(M_{\mathrm{n}}{ }^{\mathrm{PS}}=48.0 \mathrm{~kg}\right.$ $\left.\mathrm{mol}^{-1}, M_{\mathrm{n}}{ }^{\mathrm{P} 4 \mathrm{VP}}=20.3 \mathrm{~kg} \mathrm{~mol}^{-1}, M_{\mathrm{w}} / M_{\mathrm{n}}=1.13\right)$ and $\mathbf{L} 2\left(M_{\mathrm{n}}^{\mathrm{PS}}=17\right.$ $\left.\mathrm{kg} \mathrm{mol}^{-1}, M_{\mathrm{n}}{ }^{\mathrm{P} 4 \mathrm{VP}}=49 \mathrm{~kg} \mathrm{~mol}^{-1}, M_{\mathrm{w}} / M_{\mathrm{n}}=1.15\right)$, respectively. The volume fraction of the PS blocks $\left(f^{\mathrm{PS}}\right)$ of $\mathbf{L 1}$ and $\mathbf{L 2}$ are 0.70 and 0.26 , respectively. [6,6]-Phenyl- $\mathrm{C}_{61}$-butyric acid methyl ester (PCBM) was obtained from Nano-C Inc. Chloroform and methanol were purchased from Acros Organics (Geel, Belgium). All the chemicals were used as received and without further purification.

\subsection{Characterization}

The UV-Vis spectra were obtained from the solution-state with a Hitachi U4100 UV-Vis-NIR spectrophotometer at room temperature. Electrochemistry was performed with a CHI 611B electrochemical analyzer. Cyclic voltammetry $(\mathrm{CV})$ was performed with the use of a three-electrode cell in which ITO was used as the working electrode and platinum wire was used as the auxiliary electrode. All cell potentials were taken with the use of a homemade $\mathrm{Ag} / \mathrm{AgCl}, \mathrm{KCl}$ (sat.) reference electrode. The thickness of the polymer film was measured with a Microfigure Measuring Instrument (Surfcorder ET3000, Kosaka Laboratory Ltd).

Atomic force microscopy (AFM) measurements were obtained with a NanoScope IIIa AFM (Digital Instruments, Santa Barbara, CA) at room temperature. Commercial silicon cantilevers (Nanosensors, Germany) with typical spring constants of 21-78 $\mathrm{N} \mathrm{m}^{-1}$ were used to operate the transmission electron microscope (TEM). The TEM images were obtained using a JEOL JEM1230 TEM instrument operating at a voltage of $50 \mathrm{kV}$ with a Gatam dual vision CCD camera. The samples for TEM measurements were prepared by being spin-coated onto a $\mathrm{NaCl}$ substrate, and put into deionized water. The polymer composite films floated on the surface of the water and were placed onto 200-mesh copper grids. Small-angle X-ray scattering patterns were collected by X-ray scattering in a grazing-incidence geometry (Nano-Viewer, Rigaku) that includes a two-dimensional (2D) area detector (Rigaku, 100K PILATUS). The instrument is equipped with a $31 \mathrm{~kW} \mathrm{~mm}^{-2}$ generator (rotating anode X-ray source with a $\mathrm{Cu} \mathrm{K} \alpha$ radiation of $\lambda=0.154 \mathrm{~nm}$ ). The scattering vector, $q(q=4 \pi / \lambda \sin \theta)$, along with the scattering angles $\theta$ in these patterns were calibrated using silver behenate. The thin films were mounted on a $z$-axis goniometer. The angle of incidence of each X-ray beam was $\alpha_{\mathrm{i}}=0.2^{\circ}$, which is between the critical angles of the PS- $b$-P4VP films and the silicon substrates $\left(\alpha_{\mathrm{c}, \mathrm{f}}\right.$ and $\left.\alpha_{\mathrm{c}, \mathrm{s}}\right)$.

\subsection{Fabrication and measurement of the memory devices}

The memory device was fabricated with a configuration of ITO/ BCP composite/Al. The ITO glass was pre-cleaned by ultrasonication with water, acetone, and isopropanol, each for 30 min. $8 \mathrm{mg} \mathrm{ml}^{-1}$ of $\mathbf{L} \mathbf{1}$ or $\mathbf{L} 2$ containing $0-10 \%$ PCBM (relative to the weight of PS- $b$-P4VP) in chloroform were prepared and stirred for 2 weeks to form homogenous aging solutions. Thereafter, solutions were filtered through a PTFE membrane syringe filter with a $0.20 \mu \mathrm{m}$ pore size, and were further spincoated at $800 \mathrm{rpm}$ for $60 \mathrm{~s}$. Composite thin film samples were dried under vacuum for $2 \mathrm{~h}$ to remove the residual solvents. For solvent annealing, the fresh thin films were further annealed in saturated vapors of the appropriate solvent (chloroform for $\mathbf{L} 1$ and methanol for L2) for reorientation and enhancing the longrange order of the nanodomains. The solvent vapor treatment of the composite thin films was accomplished by enclosing the substrate in an inverted dish with a $50 \mathrm{ml}$ beaker containing the specific solvent at room temperature on which a weight of $\sim 2 \mathrm{~kg}$ was loaded on top of the dish. The samples were removed from the dishes after a predetermined time ( 3 days for $\mathbf{L} \mathbf{1}$ or 2 days for L2 composites), and then completely dried in an ambient atmosphere. Finally, a $300 \mathrm{~nm}$-thick Al top electrode $(0.5 \times 0.5$ $\mathrm{mm}^{2}$ in size) was thermally evaporated through a shadow mask. The electrical characterization of the memory device was performed by a Keithley 4200-SCS semiconductor parameter 
analyzer equipped with a Keithley 4205-PG2 arbitrary waveform pulse generator. All of the electronic measurements were performed in a $\mathrm{N}_{2}$-filled glove box.

\section{Results and discussion}

\subsection{Optical properties}

Upon aging, the solutions of the PS- $b$-P4VP:PCBM mixtures displayed a brown color in Fig. S1 (ESI†). Fig. 1 shows the optical absorption spectra of the PCBM:L1 and L2 mixtures in chloroform. As the PCBM content is increased from $0 \%$ to $5 \%$ and finally to $10 \%$, the absorption intensity increases in the range of $450-550 \mathrm{~nm}$. It implies the presence of induced supramolecular charge-transfer interactions between the PCBM and pyridine units of the BCP, which is similar to that reported in the literature. ${ }^{47}$ The optical band gaps of $\mathbf{L 1}$ and $\mathbf{L 2}$ estimated from the absorption edges are 4.49 and $4.51 \mathrm{eV}$, respectively.

\subsection{Thin film morphologies}

The thin film morphologies of BCP are profoundly influenced by various parameters, such as the block ratio, the strength of the interactions at the substrate interface, and surface fields of variable strength at a free surface. Here, BCP can be viewed as a template; therefore, the dispersion of the fullerene derivatives in the microphase-separated morphologies is controlled by the physical interactions after mixing. The asymmetric PS- $b$-P4VP BCP (L1 and L2) and PCBM loading ratio need to be considered to determine the morphological effect of the hydrophilic substrate which is analyzed by AFM and TEM. Fig. S3 and S4 in the ESI $\dagger$ show the AFM topographies and TEM images of the PCBM:L1 or $\mathbf{L 2}$ as-spun films with various compositions $(0 \%$, $5 \%$, and $10 \%$ ). The $\mathbf{L 1}$ pristine film displays a disordered dimpletype micellar structure with polar P4VP as the core and nonpolar PS as the corona which is due to the chloroform solvent being slightly selective, as it is good for PS but slightly poor for $\operatorname{P4VP}\left(\chi_{\text {PS-chloroform: }} 0.35 ; \chi_{\text {P4VP-chloroform: }}\right.$ 0.67). ${ }^{48,49}$ However, as PCBM is added into the polymer film, the dimple-like nanostructure clearly transforms into a more anomalous spherical shape for the PCBM:L1 thin film. By comparison, the spin-

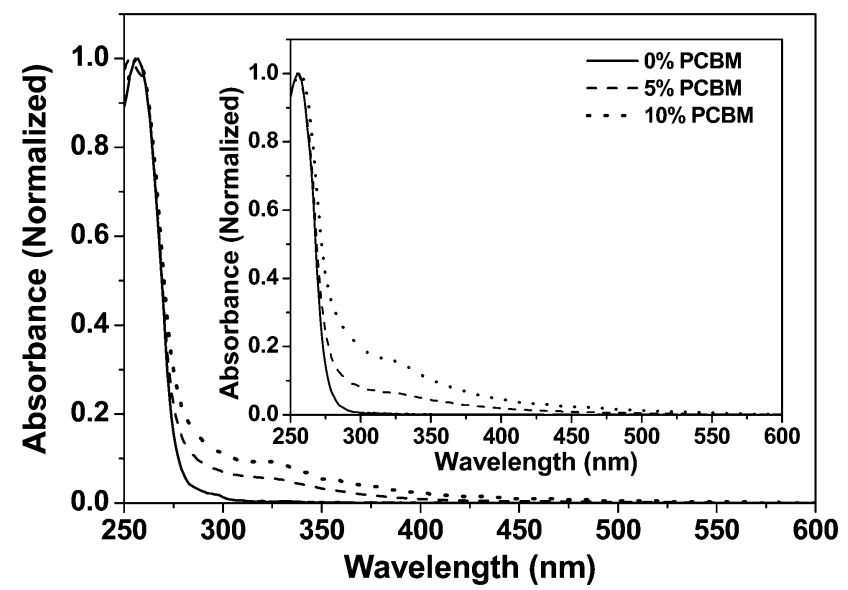

Fig. 1 Optical adsorption spectra of PCBM:L1 and PCBM:L2 (inset figure) in chloroform. coated L2 thin film without PCBM exhibits disordered wormlike nanodomains. The aggregated dark phase has mainly arisen from the P4VP domain with a longer chain length. The wormlike PCBM:L2 micelles are carefully identified by TEM, in which the selective distribution of PCBM within the P4VP domain enhances the contrast between the PS and P4VP blocks due to the high electron density of PCBM. The TEM image of the PCBM: L2 thin film exhibits more distorted micelles. The kineticallydriven disordered aggregated PCBM dispersion near the P4VP cores obtained upon fast evaporation of the solvent are not thermodynamically stable and thus could not obtain a stable morphology in sufficient time.

To achieve long-range well-ordered composite nanostructures, fresh monolayered micelle films were further annealed under the saturated vapor of an appropriate solvent. ${ }^{40-46}$ As expected, a remarkable variation in morphologies resulting from the enhancement on the development of the nanodomains with a vertical orientation (L1) or parallel orientation (L2) was observed. ${ }^{41,45,46}$ Fig. 2 and Fig. 3 show the morphologies in thin films obtained from the PCBM:L1 and L2 composites under chloroform and methanol annealing, respectively, where the PCBM loading ratios range from $0 \%$ to $5 \%$ and $10 \%$. The PCBM:L1 thin films after chloroform vapor-annealing assemble
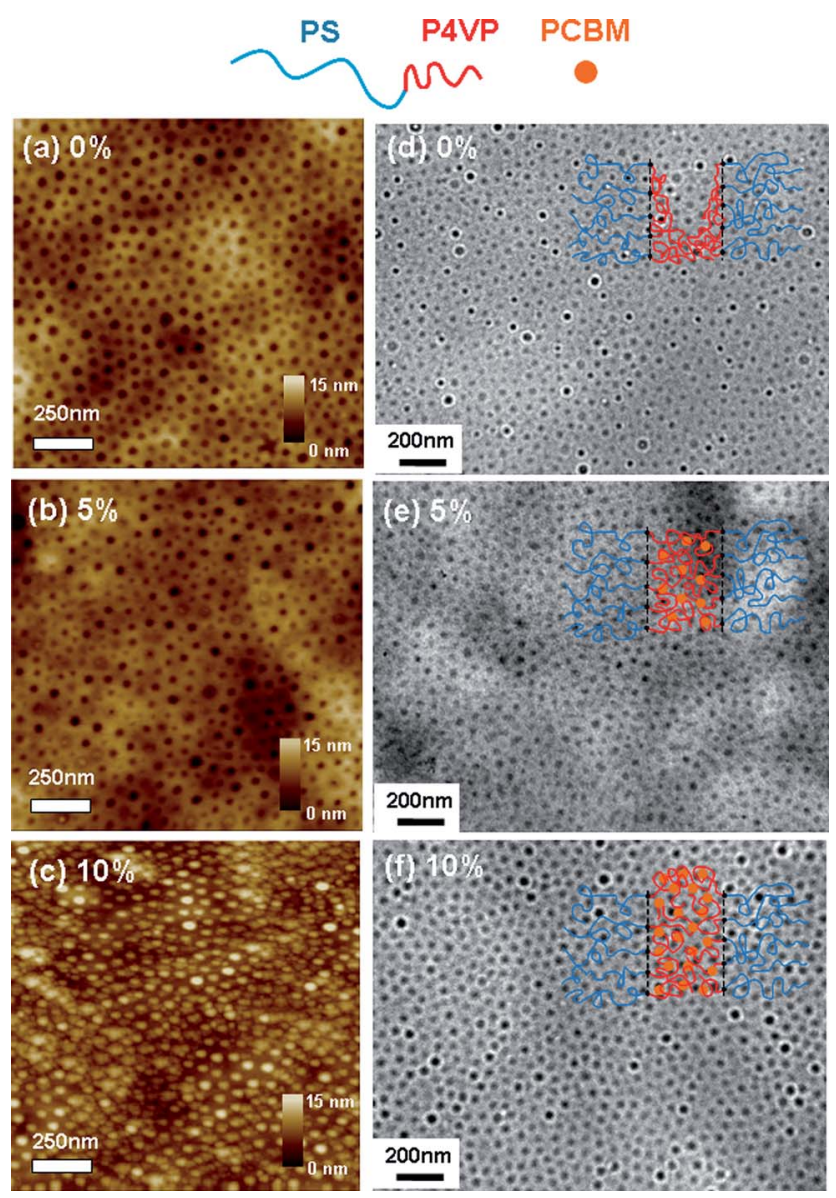

Fig. 2 AFM (a-c) height images, TEM (d-f) plane view images and schematic illustrations of the L1:PCBM composite thin films with solvent annealing under exposure to saturated chloroform vapor for 3 days. The content of PCBM: (a,d) $0 \%$, (b,e) $5 \%$ and (c,f) $10 \%$. 


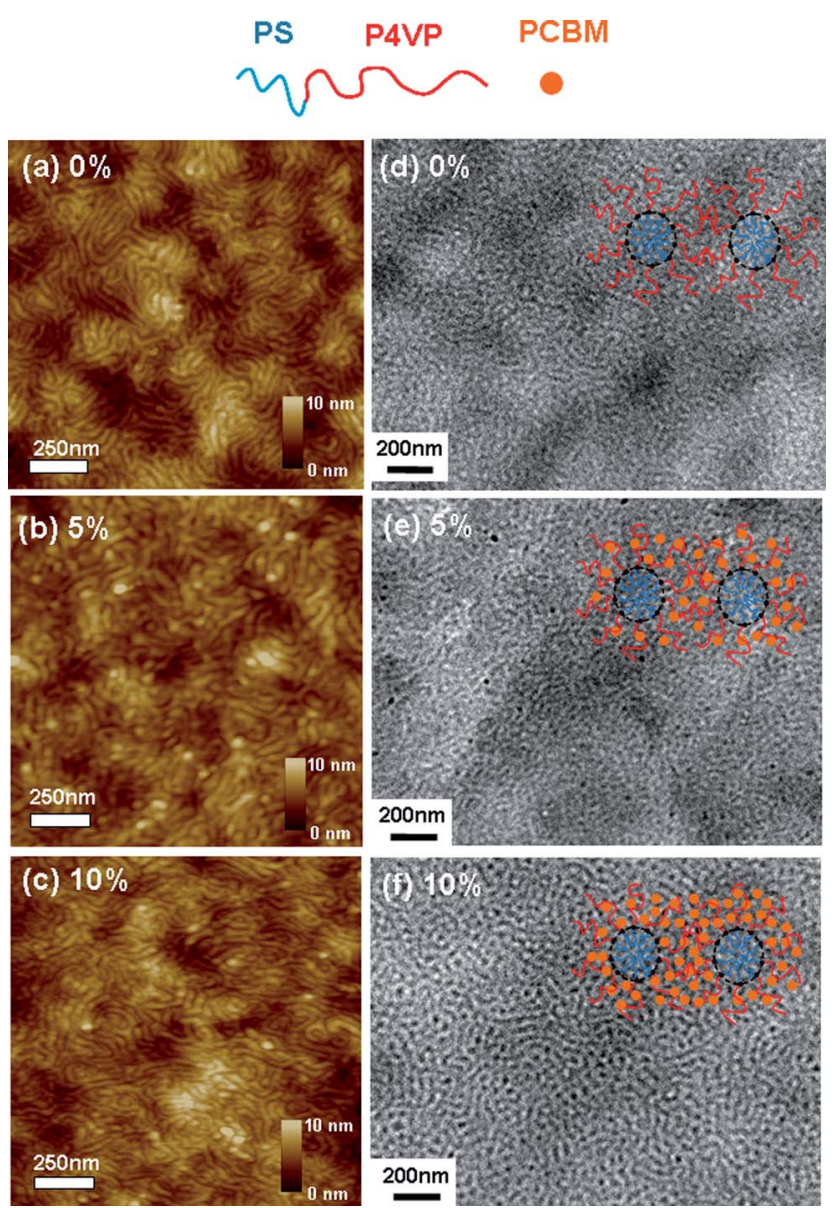

Fig. 3 AFM (a-c) height images, TEM (d-f) plane view images and schematic illustrations of L2:PCBM composite thin films with solvent annealing under exposure to saturated methanol vapor for 2 days. The content of PCBM: (a,d) $0 \%$, (b,e) $5 \%$ and (c,f) $10 \%$.

into cylinders oriented normally to the substrate with a spacing between the average adjacent core center of $c a$. $60 \mathrm{~nm}$ and pore diameter of $35 \mathrm{~nm} .{ }^{41}$ However, the disordered spherical or wormlike micelles turn into cylindrical micelles with a high aspect ratio aligned parallel to the substrate with further methanol annealing on the PCBM:L2 composite film. ${ }^{41,45,46}$ In the case of the PCBM: L1 film, when the fresh dimple-like polymer films are placed into the inverted dish containing the saturated chloroform vapor, chloroform molecules diffuse into the films and swell both the PS and P4VP chains. Therefore, solvent annealing in chloroform imparts substantial mobility to the polymer chains. It promotes the development of phase-separated nanodomains in the thin films and the homogenously selective distribution of PCBM in the P4VP domain. Note that chloroform is a slightly selective solvent for PS and thus the PS blocks form a shell around the P4VP blocks to give rise to the energetically favorable interaction with the solvent. The PCBM molecules can be easily revealed to fill the core of the cylinder (Fig. 2) since P4VP chains can attract PCBM via the supramolecular interaction, and the micro-phase separation of BCP reduces the PCBM aggregation size. Both AFM topographies and TEM micrographs are reasonably consistent, showing that the loading fillers of the cylinder cores increase remarkably with the content of PCBM. At 10\% PCBM
(Fig. 2(c)), the selective incorporation of PCBM with P4VP domains makes discrete regions appear as nano-size spotted domains protruding slightly in height relative to the continuous PS matrix. The spatial perpendicular dispersion of PCBM in the reoriented L1 matrix significantly influences the electrical properties of composite thin film.

The inside of the inverted dish containing the saturated methanol vapor is a hydrophilic environment, in which sufficient mobility is imparted to the both blocks of $\mathbf{L 2}$ (especially in the P4VP chains). It makes them self-organize to a stable cylindrical structure with a swollen P4VP corona and an expelled PS core since methanol prefers contact with P4VP rather than PS $\left(\chi_{\text {PS-methanol }}: 2.36 ; \chi_{\text {P4VP-methanol }}: 1.26\right) .{ }^{48,49} \mathbf{L 2}$ cylinders prefer to align parallel to the substrates due to the strong affinity between the P4VP chains and the substrate surface with high surface tension (Fig. 3). The strong interaction of PCBM with polar P4VP blocks makes PCBM tend to disperse in the continuous P4VP corona domains. The fused cylindrical structures can be maintained even with a higher PCBM loading ratio. The retransformed phenomenon indicates that the solvent vapor annealing approach is successfully employed in the BCP/ fullerene composite.

Under the solvent annealing conditions, the self-assembled thin film reaches an equilibrium state, in which the pristine state of L1 is comprised of discrete P4VP nanocylinders embedded within the continuous PS matrix, whereas that of $\mathbf{L 2}$ reveals a reverse morphology (i.e., PS nanocylinders are embedded within the P4VP domain). Thus, electrical information access of the well-defined storage sites could be performed on the nanoscale.

\subsection{Grazing-incident small angle X-ray scattering}

To acquire further insights into the morphologies of the thin films, we performed GISAXS measurements on the thin films at an incident angle of $0.2^{\circ}$. In-plane scan cuts were implemented on the 2D GISAXS patterns to show the 1D scattering profiles. Fig. 4(a) reveals in-plane 1D GISAXS profiles of $\mathbf{L} 1$ and PCBM: L1 composite thin films with solvent annealing in chloroform for 3 days. The in-plane 1D GISAXS profile for the pure $\mathbf{L} 1$ thin film on $\mathrm{SiO}_{x} / \mathrm{Si}$ features sharp diffraction peaks located at $q_{/ /}=$ $0.0125,0.0217$ and $0.025 \AA^{-1}$ (indicated by arrows). These features with $q_{/ /}$ratios $1: 3^{1 / 2}: 4^{1 / 2}$ are clearly discerned, indicating the formation of the vertical cylinders with a hexagonal lattice. ${ }^{50,51}$ According to the position of the principal peak, the inter-cylinder distance could be determined to be $58 \mathrm{~nm}$ for the L1 thin film. In contrast, the PCBM:L1 hybrid thin films on the $\mathrm{SiO}_{x} / \mathrm{Si}$ substrate reveal the in-plane diffraction peaks with depressed intensity and broadening, indicative of poor lateral ordering of the vertically-oriented P4VP nanocylinders. The reduced lateral ordering is resultant from the increased stretching of the P4VP chains because of the selective binding of the PCBM with the P4VP domain, evinced by a shift in the diffraction position to a smaller $q_{/ /}$. On the other hand, as more PCBM $(10 \%)$ is added, it is highly likely that the PCBM aggregates to form large clusters within the P4VP domain, giving rise to an increase in the entropic penalty of the P4VP chains. ${ }^{51}$ Therefore, small grains of nanocylinders with a large density of defects might be produced as a result of unfavorable stretching of the 
(a)

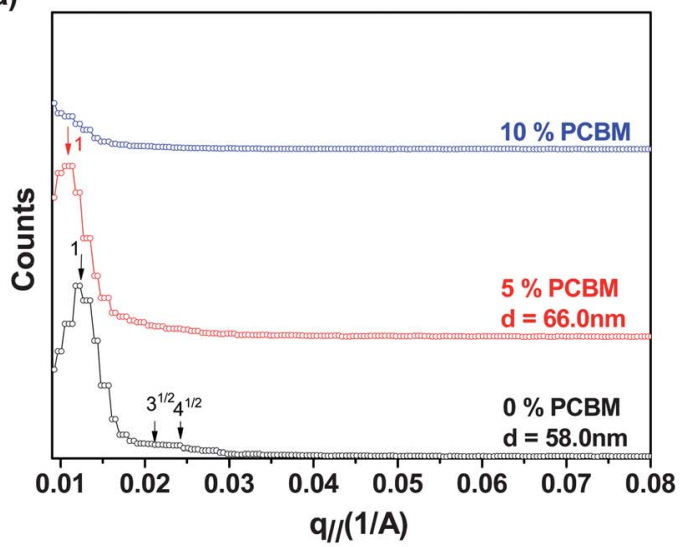

(b)

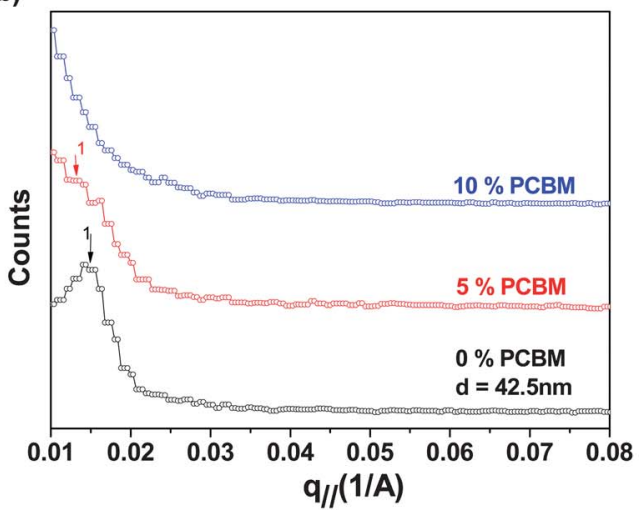

Fig. 4 GISAXS diffraction profiles (a) PCBM:L1 composite thin films with solvent annealing under exposure to saturated chloroform vapor for 3 days - perpendicularly cylindrical array; (b) PCBM:L2 composite thin films with solvent annealing under exposure of saturated methanol vapor for 2 days - horizontally cylindrical array.

polymer chains when the content of PCBM was $10 \%$. Such a phenomenon accounts for the disappearance of the higher order diffraction peaks.

As can be seen in Fig. 4(b), the $\mathbf{L 2}$ thin film on the $\mathrm{SiO}_{x} / \mathrm{Si}$ substrate only reveals a principal Bragg peak. In the previous section, the AFM and TEM images suggest that the $\mathbf{L} 2$ thin film is comprised of parallelly-oriented PS nanocylinders within a P4VP matrix. Therefore, one or two monolayers of the parallelly-oriented PS nanocylinders formed within the $\mathbf{L} 2$ thin film and contribute to the principal Bragg peak. ${ }^{52,53}$ As a result, the diffraction position is directly associated with an intercylinder spacing. In comparison with the $\mathbf{L} \mathbf{2}$ thin film, such a Bragg peak for the PCBM:L2 thin film presents the variance in the $q_{/ /}$position and diffraction intensity. Similar to the case of the PCBM:L1 composite film, the variances in the diffraction feature of the GISAXS is due to the selective swelling of the P4VP domains by PCBM.

\subsection{Memory device characteristics}

The electrical behaviors were tested by the current-voltage $(I-V)$ characteristics on the ITO/polymer composite/Al sandwiched architecture with sweeping steps of $0.1 \mathrm{~V}$. The thickness of the self-assembled L1 or L2:PCBM thin films with or without solvent annealing was adjusted to be $60-70 \mathrm{~nm}$ with a smooth surface. The loading ratio of the PCBM content in the blend solutions includes $0 \%, 3 \%, 5 \%, 7 \%$, and $10 \%$. Furthermore, each device also contained 25 cells for the statistical test of the electrical characterization. Here, the effects of PCBM content, BCP composition and solvent annealing conditions on the memory device performance were systematically characterized and analyzed.

The electrical properties and switching behaviors of the L1: PCBM composite thin films after solvent annealing are illustrated by the $I-V$ measurements shown in Fig. 5. The pure L1 based device exhibits only a low current in the range of $10^{-13}$ $10^{-10} \mathrm{~A}$ with the external applied voltage due to its insulating nature. As the loading ratio of PCBM increases, the electronic transition starts to go from insulating to the memory switching and conducting state gradually. By incorporation of a small amount of PCBM (3\%) into the L1 matrix, no obvious changes occur in the electronic properties, except for a slightly higher current response as compared to the pure $\mathbf{L} \mathbf{1}$ device and only a few memory cells show the switching transition in the electrical properties. However, most of the 5\% PCBM composite memory cells exhibit distinctly bistable electrical resistance states. The complete electrical scans of the device based on the 5\% PCBM:L1 thin film after annealing are demonstrated through three voltage sequences: 0 to $-4.0 \mathrm{~V}$ (sweep 1); 0 to $-4.0 \mathrm{~V}$ (sweep 2); 0 to $4.0 \mathrm{~V}$ (sweep 3). Initially the as-fabricated device is in the OFF state (" 0 " signal in data storage) with a current in the range of $10^{-12}$ $10^{-10} \mathrm{~A}$ as the voltage sweeps from 0 to $-1.5 \mathrm{~V}$ progressively. When the voltage increases further, an abrupt current jump occurs at a threshold voltage of $-1.5 \mathrm{~V}$, indicating that the dramatic transition from the low-conductivity OFF state to the high-conductivity ON state ("1" signal in data storage). This electronic transition in the first sweep serves as the "writing" process. The current is maintained in the $\mathrm{ON}$ state for the subsequent negative sweep from 0 to $-4.0 \mathrm{~V}$ (sweep 2) and positive sweep from 0 to $4.0 \mathrm{~V}$ (sweep 3). This device can not return to the OFF state even after turning off the power (at least $1 \mathrm{~h}$ ) or applying a reverse bias, indicating a non-volatile writeonce-read-many-times (WORM) memory behavior with a high $\mathrm{ON} / \mathrm{OFF}$ current ratio of $10^{6}$ at $-1.0 \mathrm{~V}$. The stability of the memory device against switching time with a constant voltage

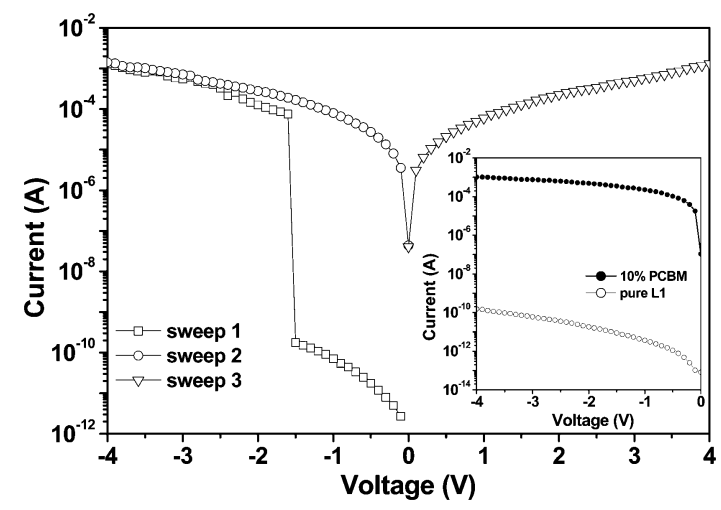

Fig. $5 I-V$ characteristics of the ITO/5\% PCBM:L1/Al device with solvent annealing. The inserted figure shows the characteristics of the $0 \%$ and 10\% PCBM:L1 device. 
bias for long periods or continuous pulse voltage stress with a pulse period of $2 \mu$ s and a pulse width of $1 \mu$ s was also evaluated under the same conditions. Both $\mathrm{ON}$ and OFF state currents of the WORM device can be sustained for up to $2.8 \mathrm{~h}$ and $10^{8}$ continuous read pulses of $-1 \mathrm{~V}$, as shown in Fig. S5 of ESI†. From this result of excellent stability of the WORM memory device, the archive is supposed to be reliably kept and permanently stored. Besides, some of the device cells with the $7 \%$ PCBM content also show a similar bistable WORM switching behavior and others exhibit the conductor behavior. However, the 10\% PCBM:L1 device exhibits only a single conduction characteristic due to the high conductivity of the large PCBM content.

In the case of the PCBM:L2 device, a similar trend in the electrical properties changes from insulator to memory switching and conductor as the PCBM content increases from $0 \%$ to $10 \%$, as shown in Fig. 6 and Fig. S6 of ESI†. The consecutive sweep cycles of the 5\% PCBM:L2 device after solvent annealing have demonstrated bistable conductivity states (Fig. 6). As a negative bias voltage is applied, the current of the memory cell suddenly increases to the higher conductance state at a threshold voltage of $-2.7 \mathrm{~V}$ with an ON/OFF current ratio of $10^{6}$. After reading the $\mathrm{ON}$ state in the negative sweep (sweep 2), the subsequent application of the third sweep is conducted after turning off the power for about $5 \mathrm{~min}$. It can be reprogrammed from the OFF state to the $\mathrm{ON}$ state when the voltage bias increases to $-2.6 \mathrm{~V}$ (sweep 3) and the current is also kept in the ON state (sweep 4). For the following repeated sweep cycle, the $I-V$ curves suggest that the ON state can be maintained for an approximate period of 5-8 min after the removal of power and would gradually relax back to the OFF state. Therefore, the short retention ability based on the electrical switching concludes the volatile nature of a static random access memory (SRAM) on most of the ITO/5\% PCBM: L2/Al cells after solvent annealing. In addition, the unstable $\mathrm{ON}$ state of the volatile memory device can be also retained by a refreshing voltage pulse of $1.0 \mathrm{~V}$ with a $1 \mathrm{~ms}$ duration every $30 \mathrm{~s}$ (named as refresh trace). The long-term stability and endurance are also evaluated from the retention time test under the voltage stress and stimulus effect of the read pulse, as shown in Fig. S7 of ESI $\dagger$. Note that the existence of the PCBM molecules in polymer

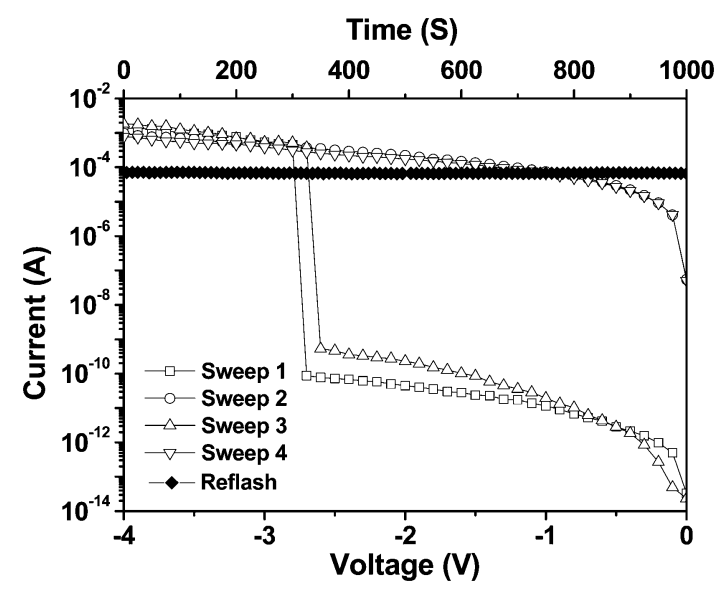

Fig. $6 I-V$ characteristics of the ITO/5\% PCBM:L2/Al device with solvent annealing. matrix obviously determines the electrical properties both in the PCBM:L1 and PCBM:L2 polymer composite device.

To classify the solvent annealing effect of the composite thin film on the switching performance, the statistical data including the threshold voltage (Fig. 7 and Fig. S8 of ESI $\dagger$ ), the switching probability (Fig. S9 and S10 of ESI $\dagger$ ) and the ON or OFF current (Fig. 8 and Fig. S11 of ESI $\dagger$ ) extracted from the randomly selected 25 cells in the single memory device were analyzed in detail. For the PCBM:L1 composite film without solvent annealing, the $1(3 \%$ PCBM) and 12 device cells $(5 \%$ PCBM) devices are able to switch to the ON state as a WORMlike memory device, and the others just work as insulators (Fig. S9 of ESI $\dagger$ ). The operation of switching on the bistable WORM cells of the 7\% PCBM:L1 device increases to 16 cells, and others almost exhibit a conducting behavior. However, the device based on the $10 \%$ PCBM only displays a high conductance state without any significant switching effect. The electronic behaviors of the PCBM:L1 composite films with solvent annealing were also investigated. There are 5, 22, and 9 out of 25 cells capable of the switching behavior for the devices containing $3 \%, 5 \%$ and $7 \%$ PCBM (Fig. S9 of ESI $\dagger$ ). Of the devices containing the PCBM composites, it is found that the devices with solvent annealing have significantly improved switchable cells compared to the non-annealed ones. Among all, the 5\% PCBM: L1 device with solvent annealing is found to exhibit noticeable switching WORM memory behavior. Similar switching phenomena were observed in the PCBM:L2 composite device (Fig. S10 of ESI $\dagger$ ) although it exhibits a volatile switching behavior. In addition, the excellent switching performance is based on the annealed 5\% PCBM:L2 composite device with 22 switchable cells out of total 25 cells. Apparent electrical transition and better memory switching effect are expected for composite thin films with solvent annealing under enough numbers of statistical data. Again, the PCBM molecules directly play an important role in the operation of the fabricated composite memory device.

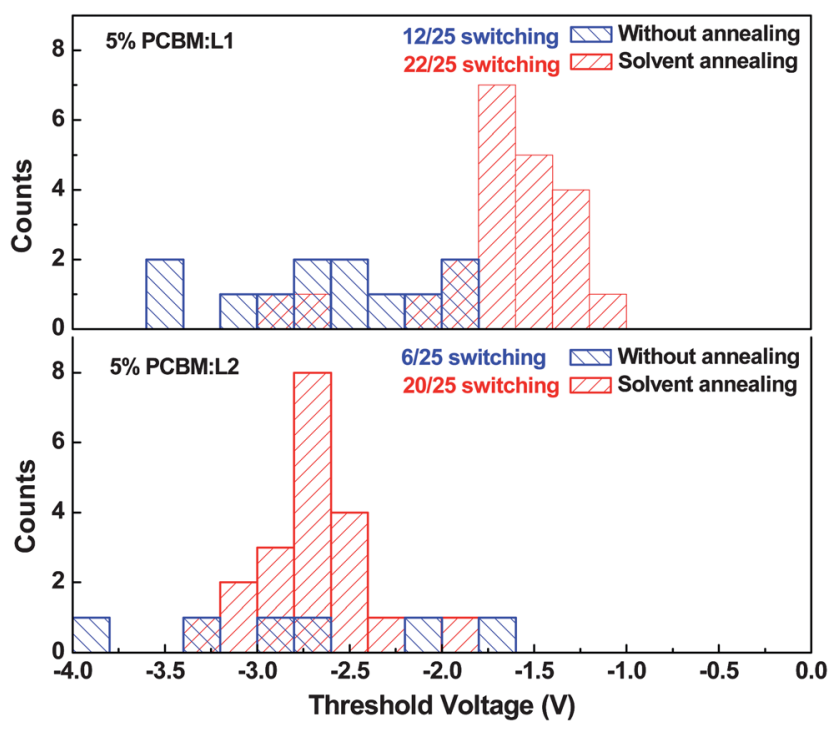

Fig. 7 Threshold voltage distributions of the ITO/5\% PCBM:L1 or L2/ $\mathrm{Al}$ device with and without solvent annealing. 
(a)

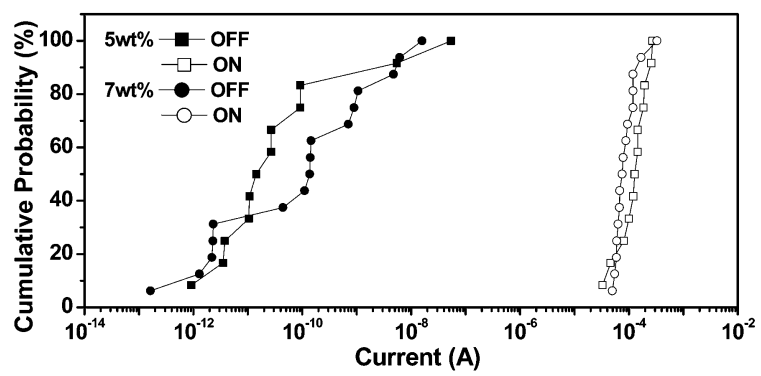

(b)

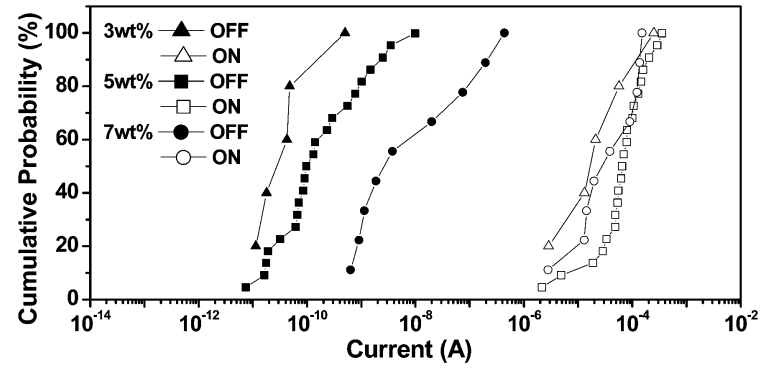

Fig. 8 Cumulative probability data (OFF and $\mathrm{ON}$ current at $-1.0 \mathrm{~V})$ for the PCBM:L1 composite system (a) without solvent annealing (b) with solvent annealing treatment.

Furthermore, the statistical variation of the threshold voltage and the current distribution of the ON and OFF states in each switchable device obtained from the voltage sweep of the individual cells are considered to demonstrate the switching condition systematically. For the devices without solvent annealing, a relatively wide distribution of the threshold voltage can be observed in both the PCBM:L1 and $\mathbf{L} 2$ composite devices (blue bar in Fig. 7 and Fig. S8 of ESI $†$ ). For example, the threshold voltage of 5\% PCBM:L1 is located in the voltage range of -1.8 to $-3.5 \mathrm{~V}$. The variation problem is due to the non-uniform distribution of the aggregated PCBM in the vertical direction. However, for the devices with solvent annealing (red bar in Fig. 7 and Fig. S8 of ESI $\dagger$ ), the threshold voltage of the switching device after solvent annealing has a narrower distribution than that containing the same PCBM content without annealing (especially for 5\% PCBM), indicating that the individual cells can be switched more uniformly. Besides, the turn-ON voltage would obviously be decreased as the PCBM concentration increases. The minor fluctuation in the electrical properties such as from moisture and oxidation should be excluded since all the devices are prepared and measured inside the glove box. Therefore, the threshold voltage should be strongly associated with the PCBM additive loading and dispersion as well.

Fig. 8 shows the effect of the PCBM content of the L1 composite on the OFF and ON current distribution of the operating cells ( read at $1 \mathrm{~V}$ ). It shows that the $\mathrm{ON}$ current is almost located in the similar range of $10^{-5}-10^{-4} \mathrm{~A}$ and not sensitive to the PCBM content. However, a higher OFF current can be observed if more PCBM is added into the composite thin film. As a result, 3\%, 5\% and 7\% PCBM devices, with solvent annealing, exhibit an ON/OFF ratio of about $10^{6}, 10^{5}-10^{6}$ and $10^{4}$, respectively, and thus the device with a smaller PCBM content has a higher ON/OFF ratio. In addition, the OFF state of the device without solvent annealing is distributed over a wider current range than that of the device after annealing, probably due to the dispersed morphology of the PCBM molecules. From the analysis of the $I-V$ experiments, it can be seen that as the PCBM loading ratio gets higher, the threshold voltage decreases and the OFF state current increases. Among the tested devices, 5\% PCBM:L1 or the $\mathbf{L} 2$ composite device, with solvent annealing, shows excellent memory performance with the highest yields on switching operation, narrow distribution on threshold voltages and reasonable separation between the $\mathrm{ON}$ and $\mathrm{OFF}$ states for a low-misleading rate. Suitable PCBM content and solvent vapor-induced self-assembled morphology can be recognized to apparently form the memory switching channel.

\subsection{Mechanism of memory switching behavior}

The obtained $I-V$ characteristics for the $5 \%$ PCBM:L1 WORM and PCBM:L2 SRAM devices were further analyzed by fitting appropriate conductance models, as shown in Fig. 9 and Fig. S12 (ESI $\dagger$ ). With a device configuration of ITO/BCP:PCBM/Al, large band gap PS- $b$-P4VP BCP may act as carrier blocking moieties and the low-lying LUMO of the PCBM compared to the $\mathrm{BCP}$, can serve as the electron trapping sites embedded in the polymer matrix. The schematic diagrams of the electronic structures corresponding to the operating mechanism are shown
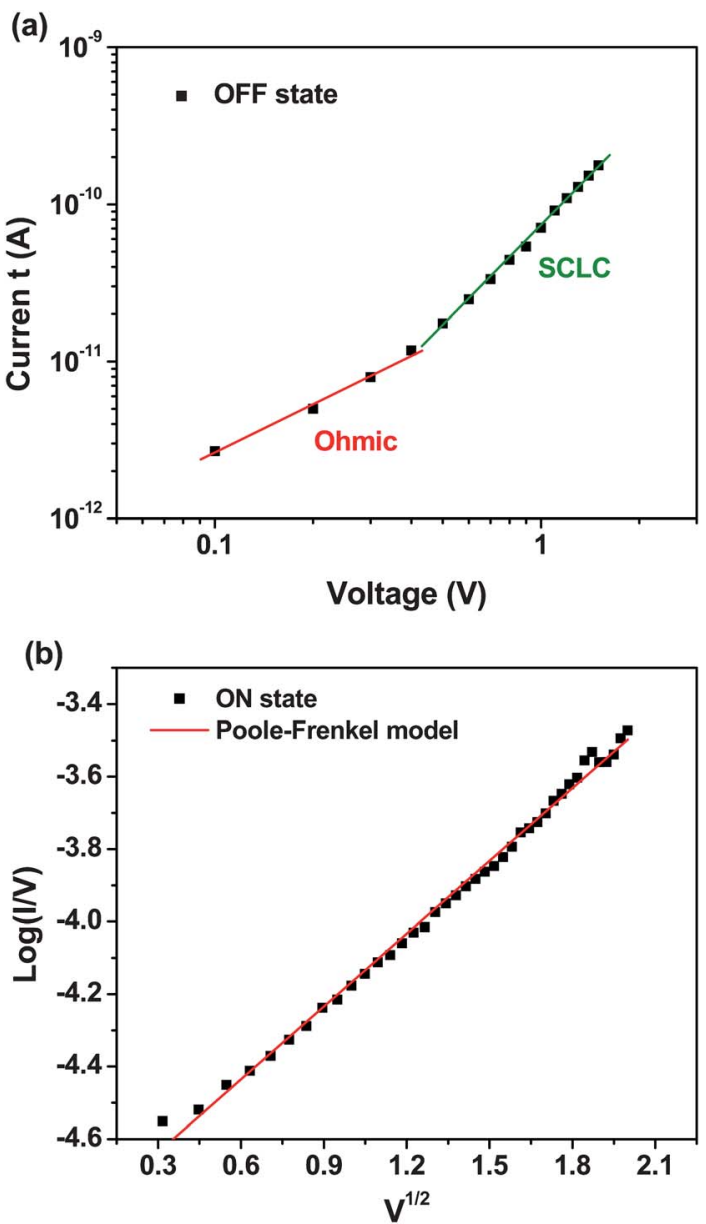

Fig. 9 Analysis of $I-V$ characteristics of (a) OFF state and (b) ON state based on the ITO/5\% PCBM:L1/Al device. 
in Fig. S13 of ESI. $\uparrow$ When the negative voltage is applied, electrons are injected into the composite film, trapped by PCBM and then escape from the isolated PCBM trapping islands through a hopping process if sufficient energy can be acquired. At low voltages (from 0 to $0.4 \mathrm{~V}$ in first sweep), the current increases linearly with the increase of the voltage bias, indicating that the current response is dominated by an Ohmic model. This is attributed to the thermally generated free electrons at the interface or in the composite layer from the electron injection. With a continuous increase of the voltage bias, the current shows that the voltage square dependence for the voltage between $0.4 \mathrm{~V}$ to the threshold voltage is approximately correlated to the spacecharge-limited-current (SCLC). ${ }^{54,55}$ The space-charge accumulates with an increasing voltage $(>0.4 \mathrm{~V})$, and is controlled through thermally active hopping conductions. The power-law relationship is quite similar to the charge-transport model of quantum dots or nanoparticles, or conjugated polymers separated by the tunneling barriers. ${ }^{18,56,57}$ After exceeding the threshold voltage (achievement of the writing process), the injected electrons from the $\mathrm{Al}$ electrode can possibly capture and fill the trapping sites. The electrons are able to flow effectively and continuously in the composite film and the current increases sharply around six orders of magnitude to a high conductive state. The electrical switching behavior results from the efficient hopping of charge carriers in the neighboring PCBM molecules. In the ON state, after electrical switching, the plot of $\log (I / V)$ versus $V^{1 / 2}$ shows a linear characteristic (Fig. 9(b)). It suggests that the Poole-Frenkel emission is the dominant charge-transport process of the $\mathrm{ON}$ state, with electrically charged defects through the bulk materials. ${ }^{27}$

The loading ratio of PCBM in the $\mathrm{BCP}$ matrix allows the device to manipulate the threshold voltage and ON/OFF current ratio, since the separation of the PCBM domain can be controlled through the PCBM distribution in the microphaseseparated $\mathrm{BCP}$ matrix. ${ }^{28,29}$ Only a few device cells can be switched in the 3\% PCBM:L1 or $\mathbf{L 2}$ composite due to the large separation between PCBM domains, which prevents the charge carriers from hopping. However, the $5 \%$ or $7 \%$ PCBM composite devices exhibit smaller threshold voltages and ON/OFF ratios (higher OFF state current) than the $3 \%$ device. The decrease in distance between the isolated PCBM molecules can easily overcome the percolation threshold of the trapping sites for the effective charge carrier transport. The energy barrier for chargetransport decreases with the increase in the PCBM content of the composites, and more charges in the memory layer can be captured by PCBM molecules. For the device containing $10 \%$ PCBM, the close stacking PCBM can form a continuous network in the active layer. It may lead to electrically effective verticalconnected channels (ohmic conduction) between the top and bottom electrodes from the aggregated PCBM molecules and short-circuit the devices directly.

The self-assembled nanostructures of the $\mathbf{L} \mathbf{1}$ and $\mathbf{L} \mathbf{2}$ composite systems, with or without solvent annealing, have a great influence on the memory performances, such as memory switching percentage, threshold voltage distribution and volatility of the data storage. Optimized PCBM distribution is especially an important factor for obtaining two-terminal bistable composite memory devices. For the $\mathbf{L} \mathbf{1}$ composite system, the non-annealed morphology is based on dimple-like micelles and the annealed nanostructure shows perpendicular cylinders with a P4VP: PCBM core and a PS corona in a hexagonal array. For the PCBM:L2 composite system, the non-annealed film only shows a disorder of the aggregated phases, and horizontal cylinders with a PS core and a P4VP:PCBM corona can be obtained after annealing. The switching characteristic parameters of the memory devices critically depend on the properties of the active memory materials and relative interfaces. Both the L1 and L2: PCBM composite thin films, with solvent annealing, exhibit narrower threshold voltage distributions and higher effective switching percentages than the non-annealed disordered film, indicating that composite films with specific nanostructures obtain well-dispersed PCBM in the P4VP domains to form electron transporting channels, as shown in Fig. 10. Particularly, the volume fraction of each block can be used to tune the different types of charge transport architecture. Also, the inter PCBM spacing also depends on the self-organized BCP at the molecular level. Therefore, the results of the high bistability effects could be achieved in terms of well distribution on functional molecules guided by the ordered BCP host matrix. It is worthy to note that the distinct difference in threshold voltage and volatility of the data storage between L1 and L2:PCBM annealed composite films also arise from the morphology effect. Due to the interaction between PCBM and pyridine, PCBM molecules are mainly dispersed in the core of $\mathbf{L} \mathbf{1}$ and corona of L2, and the longer P4VP block in L2 gives a larger proportion of the P4VP region in the thin film. Thus, the distance between the neighboring PCBM domains in $\mathbf{L} 1$ is probably much closer than $\mathbf{L 2}$ for the same PCBM content. As a result, the L2:PCBM film needs to obtain a stronger external electric field for hopping across the insulating matrix and charging the PCBM molecules. Thus, the average threshold voltage of the L1:PCBM device is slightly smaller than for the L2:PCBM device. The deep trapping
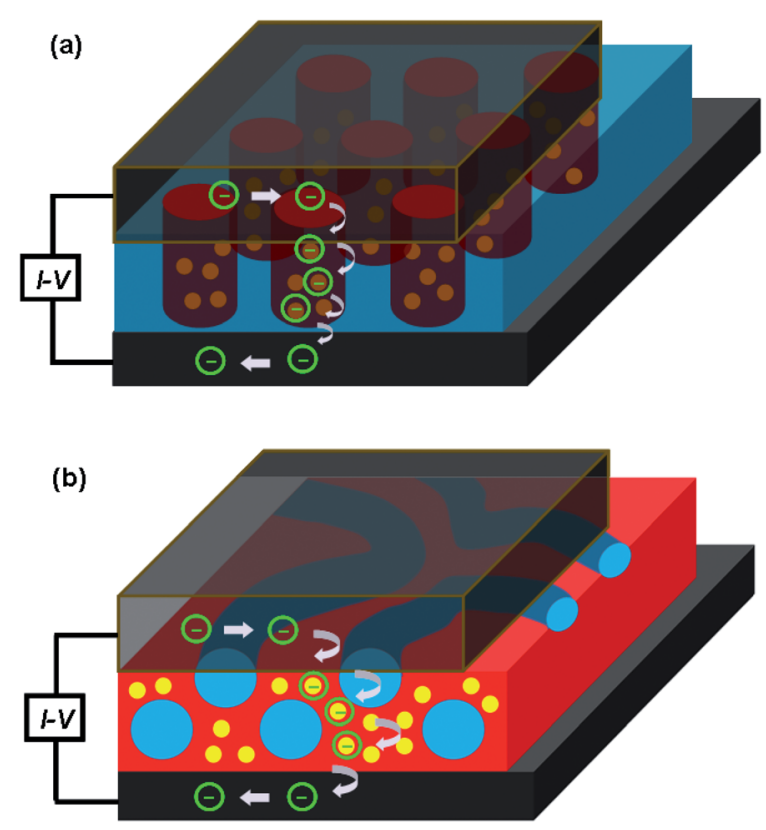

Fig. 10 Charge injection and transport behaviors in the (a) ITO/PCBM: L1/Al device and (b) the ITO/PCBM:L2/Al device after thermal annealing. 
site from the LUMO level between L1 and PCBM may not easily be emitted from by captured electrons in PCBM even through turning OFF the power or reversing the field, and the high conductance state is retained without returning to the OFF state in the observed WORM L1:PCBM device. Furthermore, for the L2:PCBM device, PCBM has more opportunities to contact with the electrode originally from the PCBM domain located on the outer side of the horizontal cylinder. The probability of the charges being able to escape is typically determined by the electrode/organic layer interface. When the power is turned off, the trapped electrons can be extracted from the PCBM to the electrode and the system returns to the original OFF state leading to the volatile nature of the L2:PCBM memory device. The remnant ON states (but still volatile) of the L2:PCBM device may be due to the delayed transfer of the trapped charge that occurs due to the L2/PCBM large trapping barriers.

In addition, the convincing switching behavior of the composite film is strongly based on the morphology- and PCBM concentration-dependent electronic properties, which rules out the possibility of an electronic transition from metal filament formation. The difference in the spatially distributed PCBM molecules has a strong relationship to the device performance. Therefore, it concludes that the electrical switching behavior is attributed to the electric field-induced space-charge formation and intrinsic traps of the composite layer in the sandwiched memory device. Stored information in discrete and specific charge trapping sites after the solvent annealing process can improve the device reliability. Controlling the physical interaction and orientation of the BCP composites offer the practical approach to make thin film morphologies with tunable nanostructures for advanced device applications.

\section{Conclusions}

We have demonstrated tunable and controllable electrical switching behavior through utilizing self-assembled PCBM:PS- $b$ P4VP composite films with a simple sandwich device architecture. The effects of interaction between the PCBM and PS- $b$-P4VP and the reorientation force led to specific thin film morphologies for electrical memory device as evinced by AFM, TEM, and GISAXS. The $I-V$ characteristics for the PCBM:PS$b$-P4VP composite device showed the electrically insulating, bistable switching or conducting effect and a strong dependence on the loading of the PCBM, block length ratio and solvent annealing conditions. Also, the memory characteristics could be controlled by the morphology of the BCP composite thin film using a different block ratio or solvent annealing. The L1:PCBM memory device showed a non-volatile WORM-like memory behavior but the L2:PCBM device exhibited a volatile SRAM nature. The spatial distributions of the PCBM domains and the ability of the charges to escape or be captured in the trapping environment determined the memory characteristics. The technique allowed for the improvement of statistical threshold voltages and ON/OFF ratios with more well-defined structures after solvent annealing. The switching mechanism, based on experimental and the fitted $I-V$ curves, was explained by the spacecharge-limited-current in the OFF state and the charge-transport-dominated Poole-Frenkel model in the ON state. Electric field induced conductance transition depended on the efficient charge transport ability with the co-existing charge-trapping environment in the composite thin film. These results demonstrated the morphology-controllable charge-trapping nanoelements for undergoing memory bistable states.

\section{Acknowledgements}

C.-L. L. gives thanks for the financial support from the Dissemination of Tenure Tracking System Program of Ministry of Education, Culture, Sports, Science and Technology-Japan. W.-C. C. acknowledges the financial support from the National Science Council of Taiwan and the Excellence Research Program of National Taiwan University. We thank Prof. J. Kido (Yamagata University) and Prof. Z. Hong (Yamagata University) for their helpful discussions on the devices. The technical assistance of Dr. J.-C Hsu on TEM measurements (National Taiwan University) and Mr. C.-Y. Kuo on the GISAXS experiment (National Central University) is highly appreciated.

\section{References}

1 Q.-D. Ling, D.-J. Liaw, C. Zhu, D. S.-H. Chan, E.-T. Kang and K.-G. Neoh, Prog. Polym. Sci., 2008, 33, 917.

2 Y. Yang, J. Ouyang, L. Ma, R. J. H. Tseng and C. W. Chu, $A d v$. Funct. Mater., 2006, 16, 1001.

3 J. C. Scott and L. D. Bozano, Adv. Mater., 2007, 19, 1452.

4 P. Heremans, G. H. Gelinck, R. Müller, K.-J. Baeg, D.-Y. Kim and Y.-Y. Noh, Chem. Mater., 2011, 23, 341.

5 S.-J. Lee, J. Mater. Chem., 2011, 21, 14097.

6 C.-L. Liu and W.-C. Chen, Polym. Chem., 2011, 2, 2169.

7 S. Song, B. Cho, T.-W. Kim, Y. Ji, M. Jo, G. Wang, M. Choe, Y. H. Kahng, H. Hwang and T. Lee, Adv. Mater., 2010, 22, 5048.

8 Q.-D. Ling, Y. Song, S.-L. Lim, E. Y.-H. Teo, Y.-P. Tan, C. Zhu, D. S. H. Chan, D.-L. Kwong, E.-T. Kang and K.-G. Neoh, Angew. Chem., Int. Ed., 2006, 45, 2947.

9 X.-D. Zhuang, Y. Chen, B.-X. Li, D.-G. Ma, B. Zhang and Y. Li, Chem. Mater., 2010, 22, 4455.

10 Y.-K. Fang, C.-L. Liu, C. Li, C.-J. Lin, R. Mezzenga and W.-C. Chen, Adv. Funct. Mater., 2010, 20, 3012

11 N.-H. You, C.-C. Chueh, C.-L. Liu, M. Ueda and W.-C. Chen, Macromolecules, 2009, 42, 4456.

12 T. Kuorosawa, C.-C. Chueh, C.-L. Liu, T. Higashihara, M. Ueda and W.-C. Chen, Macromolecules, 2010, 43, 1236.

13 C.-L. Liu, T. Kurosawa, A.-D. Yu, T. Higashihara, M. Ueda and W.-C. Chen, J. Phys. Chem. C, 2011, 115, 5930.

14 C.-L. Liu, J.-C. Hsu, W.-C. Chen, K. Sugiyama and A. Hirao, ACS Appl. Mater. Interfaces, 2009, 1, 1974.

15 Y.-K. Fang, C.-L. Liu and W.-C. Chen, J. Mater. Chem., 2011, 21, 4778.

16 Y.-K. Fang, C.-L. Liu and W.-C. Chen, Macromolecules, 2011, 44, 2604.

17 R. J. Tseng, J. Huang, J. Ouyang, R. B. Kaner and Y. Yang, Nat. Mater., 2004, 3, 918.

18 H.-T. Lin, Z. Pei and Y.-J. Chan, IEEE Electron Device Lett., 2007, 28, 569.

19 B. Cho, T.-W. Kim, M. Choe, G. Wang, S. Song and T. Lee, Org. Electron., 2009, 10, 473.

20 S. I. White, P. M. Vora, J. M. Kikawa and K. I. Winey, Adv. Funct. Mater., 2010, 21, 233.

21 C. De Rosa, F. Auriemma, R. Di Girolamo, G. P. Pepe, T. Napolitano and R. Scaldaferri, Adv. Mater., 2010, 22, 5414.

22 C. O. Baker, B. Shedd, R. J. Tseng, A. A. Martinez-Morales, C. S. Ozkan, M. Ozkan, Y. Yang and R. B. Kaner, ACS Nano, $2011,5,3469$.

23 G. Liu, Q.-D. Ling, E.-T. Kang, K.-G. Neoh, D.-J. Liaw, F.-C. Chang, C.-X. Zhu and D. S.-H. Chan, J. Appl. Phys., 2007, 102, 024502 .

24 G. Liu, Q.-D. Ling, E. Y. H. Teo, C.-X. Zhu, D. S.-H. Chan, K.-G. Neoh and E.-T. Kang, ACS Nano, 2009, 3, 1929. 
25 J. K. Baral, H. S. Majumdar, A. Laiho, H. Jiang, E. I. Kauppinen, R. H. A. Ras, J. Ruokolainen, O. Ikkala and R. Osterbacka, Nanotechnology, 2008, 19, 035203.

26 J. Liu, Z. Yin, X. Cao, F. Zhao, A. Lin, L. Xie, Q. Fan, F. Boey, H. Zhang and W. Huang, ACS Nano, 2010, 4, 3987.

27 C. W. Chu, J. Ouyang, J.-H. Tseng and Y. Yang, Adv. Mater., 2005, 17,1440 .

28 J.-C. Hsu, C.-L. Liu, W.-C. Chen, K. Sugiyama and A. Hirao, Macromol. Rapid Commun., 2011, 32, 528.

29 J.-C. Hsu, Y. Chen, T. Kakuchi and W.-C. Chen, Macromolecules, 2011, 44, 5168.

30 F. S. Bates and G. H. Fredrickson, Phys. Today, 1999, 52, 32.

31 M. Changez, N. G. Ng, C. H. Lee and J. S. Lee, Small, 2010, 6, 63.

32 I. W. Hamley, The Physics of Block Copolymers, Oxford University Press, New York, 1998.

33 T. Thurn-Albrecht, J. Schotter, G. A. Kästle, N. Emley, T. Shibauchi, L. Krusin-Elbaum, K. Guarini, C. T. Black, M. T. Tuominen and T. P. Russell, Nature, 2000, 290, 2126.

34 W. L. Leong, P. S. Lee, A. Lohani, Y. M. Lam, T. Chen, S. Zhang, A. Dodabalapur and S. G. Mhaisalkar, Adv. Mater., 2008, 20, 2325.

35 W. L. Leong, N. Mathews, S. G. Mhaisalkar, T. Chen and P. S. Lee, Appl. Phys. Lett., 2008, 93, 222908.

36 W. L. Leong, N. Mathews, S. Mhaisalkar, Y. M. Lam, T. Chen and P. S. Lee, J. Mater. Chem., 2009, 19, 7354.

37 J.-S. Lee, Y.-M. Kim, J.-H. Kwon, J. S. Sim, H. Shin, B.-H. Sohn and Q. Jia, Adv. Mater., 2011, 23, 2064.

38 C.-L. Liu, C.-H. Lin, C.-C. Kuo, S.-T. Lin and W.-C. Chen, Prog. Polym. Sci., 2011, 36, 603.

39 C. Li, J.-C. Hsu, K. Sugiyama, A. Hirao, W.-C. Chen and R. Mezzenga, Macromolecules, 2009, 42, 5793.

40 T. H. Kim, J. Huh, J. Hwang, H.-C. Kimj, S. H. Kim, B. H. Sohn and C. Park, Macromolecules, 2009, 42, 6688.
41 S. Park, J.-Y. Wang, B. Kim, W. Chen and T. P. Russell, Macromolecules, 2007, 40, 9059.

42 S. Park, B. Kim, O. Yavuzcetin, M. T. Tuominen and T. P. Russell, ACS Nano, 2008, 2, 1363.

43 S. Park, J.-Y. Wang, B. Kim, J. Xu and T. P. Russell, ACS Nano, 2008, 2, 766.

44 B. K. Kuila, E. B. Gowd and M. Stamm, Macromolecules, 2010, 43, 7713.

45 C.-Y. Chang, P.-J. Wu and Y.-S. Sun, Soft Matter, 2011, 7, 9140.

46 C.-Y. Chang, Y.-C. Lee, P.-J. Wu, J.-Y. Liu, Y.-S. Sun, and B.-T. Ko, Langmuir, in press. (DOI: 10.1021/la203114j).

47 A. Laiho, R. H. A. Ras, S. Valkama, J. Ruokolainen, R. Österbacka and O. Ikkala, Macromolecules, 2006, 39, 7648.

48 A. F. M. Barton, Chem. Rev., 1975, 75, 731.

49 J. E. Mark, Physical Properties of Polymers Handbook, AIP Press, New York, 1996.

50 S.-W. Yeh, K.-H. Wei, Y.-S. Sun, U.-S. Jeng and K. S. Liang, Macromolecules, 2003, 36, 7903.

51 S.-W. Yeh, K.-H. Wei, Y.-S. Sun, U.-S. Jeng and K. S. Liang, Macromolecules, 2005, 38, 6559.

52 Y.-S. Sun, S.-W. Chien and P.-J. Wu, Macromolecules, 2010, 43, 5016.

53 Y.-S. Sun, S.-W. Chien and J.-Y. Liou, Macromolecules, 2010, 43, 7250.

54 M. A. Lampert, P. Mark, Current Injection in Solids, Academic Press, New York, 1970.

55 M. Arif., M. Yun, S. Gangopadhyay, K. Ghosh, K. Fadiga, F. Galbrecht, U. Scherf and S. Guha, Phys. Rev. B, 2007, 75, 195202.

56 D. Y. Yun, J. K. Kwak, J. H. Jung, T. W. Kim and D. I. Son, Appl. Phys. Lett., 2009, 95, 143301.

57 K. K. Park, J. H. Jung and T. W. Kim, Appl. Phys. Lett., 2011, 98, 193301. 